\title{
Split ring resonator-based left-handed coplanar waveguide
}

F. Martín, J. Bonache, F. Falcone, M. Sorolla, and R. Marqués

Citation: Appl. Phys. Lett. 83, 4652 (2003); doi: 10.1063/1.1631392

View online: https://doi.org/10.1063/1.1631392

View Table of Contents: http://aip.scitation.org/toc/apl/83/22

Published by the American Institute of Physics

\section{Articles you may be interested in}

Split-ring resonator for use in magnetic resonance from $200-2000 \mathrm{MHz}$

Review of Scientific Instruments 52, 213 (1981); 10.1063/1.1136574

Electromagnetic resonances in individual and coupled split-ring resonators

Journal of Applied Physics 92, 2929 (2002); 10.1063/1.1497452

Electric coupling to the magnetic resonance of split ring resonators

Applied Physics Letters 84, 2943 (2004); 10.1063/1.1695439

Electrically small split ring resonator antennas

Journal of Applied Physics 101, 083104 (2007); 10.1063/1.2722232

Electric-field-coupled resonators for negative permittivity metamaterials

Applied Physics Letters 88, 041109 (2006); 10.1063/1.2166681

Split ring resonator sensors for infrared detection of single molecular monolayers

Applied Physics Letters 95, 043113 (2009); 10.1063/1.3194154
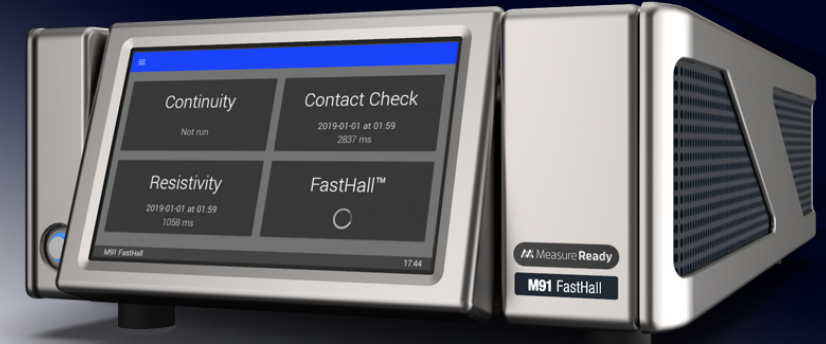

\section{M Measure Ready M91 FastHall ${ }^{\mathrm{TM}}$ Controller}

A revolutionary new instrument for complete Hall analysis 


\title{
Split ring resonator-based left-handed coplanar waveguide
}

\author{
F. Martín and J. Bonache \\ Departament d'Enginyeria Electrònica, Universitat Autònoma de Barcelona, \\ 08193 Bellaterra (Barcelona), Spain \\ F. Falcone and M. Sorolla \\ Departamento de Ingeniería Eléctrica y Electrónica, Universidad Pública de Navarra, \\ 31006 Pamplona (Navarra), Spain \\ R. Marqués \\ Departamento de Electrónica y Electromagnetismo. Facultad de Física-Universidad de Sevilla, \\ Av. Reina Mercedes s/n, 41012 Sevilla (Spain)
}

(Received 9 May 2003; accepted 8 October 2003)

\begin{abstract}
In this letter, a planar left-handed propagating medium consisting of a coplanar waveguide (CPW) inductively coupled to split ring resonators (SRR) and periodically loaded with narrow metallic wires is proposed. The wires make the structure behave as a microwave plasma with a negative effective permittivity which covers a broad frequency range. The negative permeability required to achieve left-handed wave propagation is provided by the rings in the vicinity of their resonant frequency. The result is a structure which allows negative wave propagation in a narrow frequency band. The transmission coefficient measured in a fabricated prototype device exhibits very low insertion losses in the pass band and high-frequency selectivity. Since rings are much smaller than signal wavelength at resonance and can be easily tuned, SRR-CPW-based structures are of interest for the design of very compact microwave circuits based on left handedness. (C) 2003 American Institute of Physics. [DOI: 10.1063/1.1631392]
\end{abstract}

The development of artificial materials (metamaterials) with simultaneously negative permeability and permittivity, or left-handed materials (LHMs), has been a subject of growing interest in recent years. Apart from its exotic electrodynamic properties (such as the reversal of Snell's law, Doppler effect, and Cherenkov radiation), pointed out by Veselago more than 30 years ago, ${ }^{1}$ key to this interest is the potential applicability of these metamaterials to the fabrication of radio frequency (rf) and microwave components based on left handedness. Due to negative values of effective permittivity and permeability, LHMs are negative refractive index media with antiparallel phase and group velocities. ${ }^{2,3}$ Namely, the wavevector $\mathbf{k}$ forms a left-handed triplet with the vectors $\mathbf{E}$ and $\mathbf{H}$ (the electric and magnetic field intensity) and wave fronts for propagating electromagnetic (EM) waves travel toward the source, i.e., opposite to the direction of energy flow.

Due to the absence of transparent LHMs in nature, a LHM medium has to be artificially fabricated. This can be achieved by microstructuring a material in a length scale much shorter than the wavelength of EM radiation, so that a continuous medium with effective electromagnetic properties (permittivity and permeability) is obtained. An artificial LHM operating in the microwave region was reported by Smith et al. ${ }^{4}$ The structure was fabricated by combining a periodic array of metal posts with an array of nonmagnetic split ring resonators (SRRs). Originally proposed by Pendry, ${ }^{5}$ SRRs provide a negative effective permeability in the vicinity of the resonant frequency, while metallic wires behave like a two-dimensional (2D) plasma with negative permittivity up to the plasma frequency. Left-handed wave propagation has been also demonstrated in a one-dimensional (1D) configuration consisting of a hollow metallic waveguide periodic loaded with SRRs. ${ }^{6}$ For EM propagation in the axial direction, the metallic waveguide emulates a lossless plasma whose dielectric permittivity is negative below the cutoff frequency.

The cited structures exhibit a left-handed side effect but are far from being considered as potential candidates for microwave and wireless component systems. To this end, compatibility with planar circuit technology is required. LHMs based on planar transmission lines have been recently reported. It is remarkable the work of Grbic and Eleftheriades, ${ }^{7}$ who demonstrate the existence of backward wave radiation (a characteristic analogous to reversed Cherenkov radiation) in a dual transmission line, where a host coplanar waveguide is loaded with series capacitors and shunt connected inductors. In this letter, a 1D LHM based on a coplanar waveguide inductively coupled to SRRs and periodic loaded with narrow metallic wires is presented. From the dispersion relation obtained in a lumped element equivalent circuit of the structure, it is found that phase and group velocities are antiparallel in a narrow band just above the resonant frequency of SRRs. This theoretical evidence of left-hand sided wave propagation is corroborated from full wave EM simulations and validated by measuring the transmission coefficient in a fabricated prototype device.

The proposed implementation of the left-handed structure is depicted in Fig. 1(a). This consists of a host coplanar waveguide (CPW) with a center conductor (signal strip) and two ground planes on either side separated by slots. SRRs are symmetrically placed in the back side of the substrate while thin metal wires connect the signal to the ground at those positions coincident with the center of the SRRs. Compared to the SRR-wire medium of Smith, ${ }^{4}$ or the SRRhollow metallic waveguide, ${ }^{6}$ the proposed LHM is planar and the fabrication procedure simply requires a standard mask/photoetching technique (an Arlon 250-LX-0193-43-11 


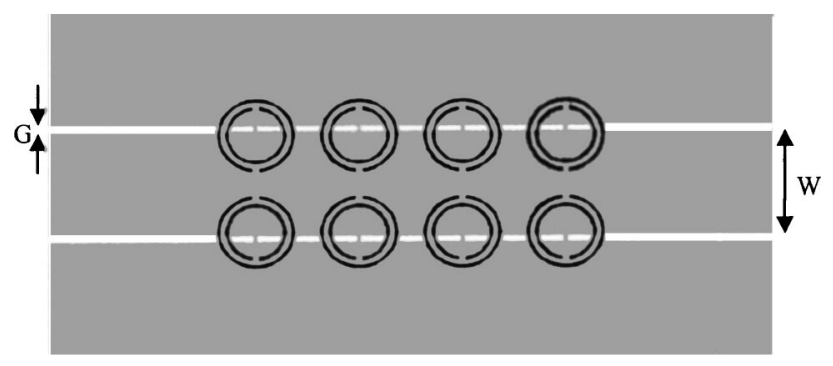

(a)

(b)

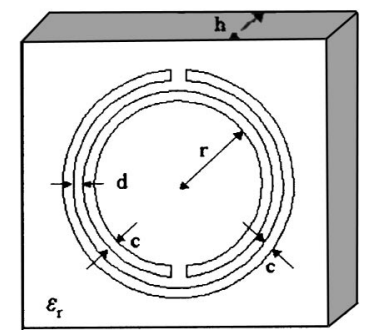

FIG. 1. (a) Layout of the SRR-based left-handed CPW structure drawn to scale. Actual device length is $3.5 \mathrm{~cm}$ and (b) geometrical parameters of the SRR.

substrate-dielectric constant $\epsilon_{r}=2.43$, thickness $h=0.49$ $\mathrm{mm}$-has been used). By placing the SRRs parallel to the $\mathrm{CPW}$, the main component of the electric field in the rings is parallel to the ring plane, while the magnetic field is polarized along the ring axis. These are necessary conditions to achieve a negative effective permeability. On the other hand, connecting wires act as the metal posts in the 2D LHM of Smith, ${ }^{4}$ and provide the effective negative permittivity which is required to obtain left-handed wave propagation in the line. In Fig. 1, the dimension of the unit cell is $\ell=5 \mathrm{~mm}$ while the center conductor and slot widths are $W=5.4 \mathrm{~mm}$ and $G=0.3 \mathrm{~mm}$, respectively. These lateral dimensions have been determined to obtain a characteristic impedance for the host line of $Z_{o}=50 \Omega$ (the impedance of measuring probes), and hence optimize transmission in the pass band. The rings have an internal radius of $r=1.3 \mathrm{~mm}$, widths of $c=0.2 \mathrm{~mm}$, and a separation of $d=0.2 \mathrm{~mm}$ [see Fig. 1(b)]. With this geometry, the resonant frequency of the rings is expected to be $f_{o}=7.7 \mathrm{GHz} .{ }^{8,9}$ Finally, the width of connecting wires is $0.2 \mathrm{~mm}$.

Let us now focus on the propagation characteristics of the proposed structure through the analysis of the dispersion relation. This is inferred from the lumped element equivalent circuit of the elemental cell [Fig. 2(a)], which is valid in the long-wavelength regime ( $\beta \ell \ll 1$, where $\beta$ is the propagation constant for guided waves). Due to symmetry, the magnetic wall concept has been used so that the equivalent circuit actually corresponds to one half the basic cell. $L$ and $C$ are the per-section inductance and capacitance of the line. These parameters model the inductance associated with the central conductor of the host CPW and the capacitive coupling between this conductor and ground planes (slot capacitance), respectively. $L_{p}$ is the equivalent inductance of connecting wires and SRRs are modeled as parallel resonant circuits (with inductance $L_{s}$ and capacitance $C_{s}$ ) which are inductively coupled to the line through a mutual inductance, $M$. To obtain left handedness, it is necessary that the shunt admittance is dominated by the inductance of the wires at the resonant frequency of the rings. This condition depends on
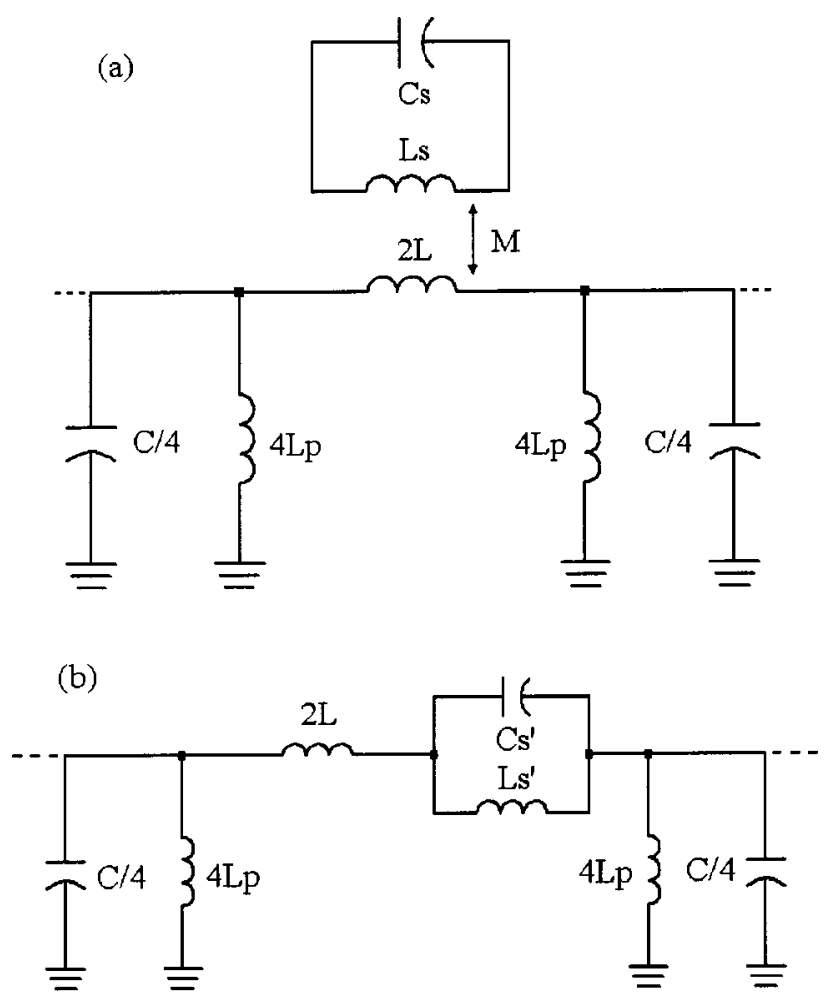

FIG. 2. (a) Lumped element equivalent circuit for the basic cell of the proposed structure and (b) simplified circuit with the series branch replaced by its equivalent impedance.

the width and distance between connecting wires and is easily achievable provided $f_{o}$ is not very high. By obtaining the equivalent impedance of the series branch, the circuit can be simplified to that shown in Fig. 2(b) (which is formally identical to that proposed in Ref. 10 for the SRR/wire medium of Smith), ${ }^{4}$ where $C_{s}^{\prime}$ and $L_{s}^{\prime}$ are given by

$$
\begin{aligned}
C_{s}^{\prime} & =\frac{L_{s}}{\omega_{o}^{2} M^{2}}, \\
L_{s}^{\prime} & =\omega_{o}^{2} M^{2} C_{s},
\end{aligned}
$$

with $\omega_{o}^{2}=1 /\left(L_{s} C_{s}\right)=1 /\left(L_{s}^{\prime} C_{s}^{\prime}\right)$. The dispersion relation for the circuit of Fig. 2(b) can be easily obtained from standard calculus: ${ }^{11}$

$$
\cos (\beta \ell)=1-\frac{L_{p} \omega-\frac{1}{C \omega}}{4 L_{p} / C}\left(2 L \omega-\frac{L_{s}^{\prime} / C_{s}^{\prime}}{L_{s}^{\prime} \omega-\frac{1}{C_{s}^{\prime} \omega}}\right) .
$$

Figure 3 shows the previous expression in a $\omega-\beta$ representation, where the scale of the vertical axis has been limited to few $\mathrm{GHz}$ to appreciate the dispersive behavior in the region of interest. The values of $L$ and $C$ have been obtained by means of a transmission line calculator; $L_{p}$ has been estimated from the simulated frequency response of the wired CPW (SRRs removed), where the plasma frequency is given by the resonator composed by $C$ and $L_{p} ;{ }^{10} M$ has been inferred from the fractional area, $F$, of the slots occupied by the rings $(M=F \cdot L)$; finally, $L_{s}$ and $C_{s}$ have been obtained according to the model described in Ref. 9. According to Fig. 3 , a narrow pass band just above the resonant frequency of 


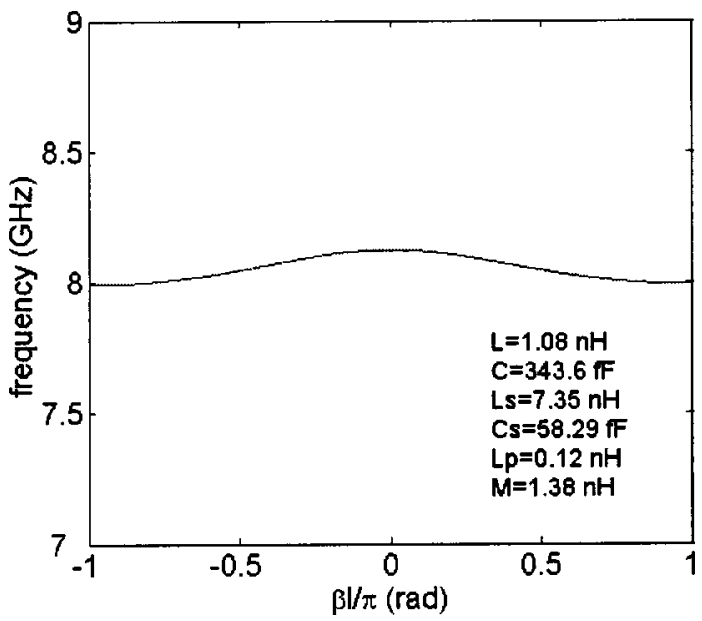

FIG. 3. Dispersion diagram for the lumped element circuit model of the SRR-CPW structure.

the rings is expected. Since $\beta$ decreases with frequency, phase and group velocities are antiparallel. Therefore, negative wave propagation occurs and the structure behaves as a narrow band LHM. If connecting wires are removed, effective permittivity is no longer negative and the pass band behavior is expected to change to a stop band. The transmission $\left(S_{21}\right)$ and reflection $\left(S_{11}\right)$ coefficients for the left-hand side structure shown in Fig. 1 and for the same structure without connecting wires, have been obtained by means of full wave EM simulation (using the commercial software CST MICROWAVE STUDIO). The results, depicted in Figs. 4 and 5, confirm the presence of a narrow pass band in the lefthanded device and a stop band behavior for the wireless structure. These structures have been fabricated in a Arlon 250-LX-0193-43-11 substrate by means of a standard mask/ photoetching technique. After soldering $3.5 \mathrm{~mm}$ connectors at the input/output ports, the transmission and reflection coefficients have been measured by means of a Agilent 8722ES vector network analyzer (the results are also depicted in Figs. 4 and 5). Reasonable agreement between experiment and simulation has been obtained. The pass band shown in Fig. 4 (which is a consequence of the simultaneous negative permittivity and permeability) is an extraordinary result for a

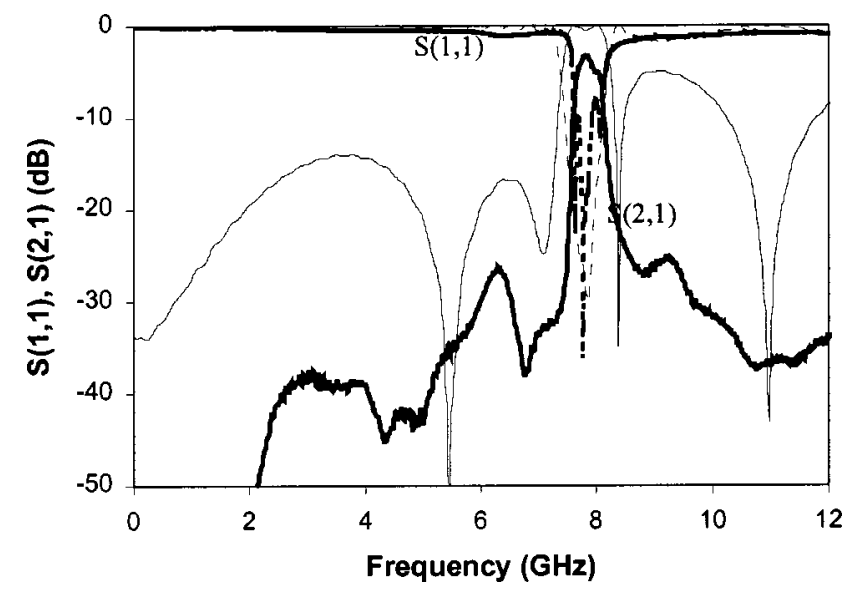

FIG. 4. Simulated (thin line) and measured (bold line) transmission $S(2,1)$ and reflection $S(1,1)$ coefficients for the left-handed SRR-CPW structure.

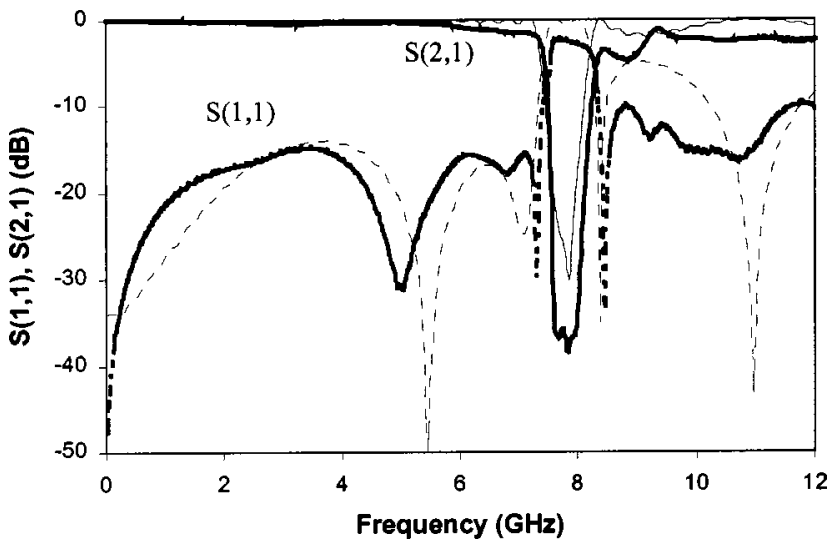

FIG. 5. Simulated (thin line) and measured (bold line) transmission $S(2,1)$ and reflection $S(1,1)$ coefficients for the SRR-CPW structure with the wires removed.

SRR-based left-handed structure: A high degree of frequency selectivity has been achieved with a transmission coefficient near $0 \mathrm{~dB}$. The slight degradation visible in the experiment is due to ohmic and dielectric losses, not accounted for in the simulations.

In conclusion, left-handed wave propagation in a CPW, coupled to split ring resonators and periodically loaded with signal-to-ground wires acting as inductors, has been demonstrated. Since negative permeability and permittivitty coexist only in the vicinity of the resonant frequency of the rings, signal propagation in the structure is limited to a narrow frequency band. This has been demonstrated in a fabricated prototype device, where the measured transmission coefficient has been indicative of negligible insertion losses in the allowed band. The proposed structure can be of interest for the fabrication of high selectivity band pass filters based on left handedness. Compatibility with monolithic microwave integrated circuit or printed circuit board fabrication technologies as well as compactness (derived from the fact that ring dimensions are electrically small), are other key aspects that lead us to think about the application of these structures to the design of miniaturized microwave and millimeter wave circuits.

This work has been supported by DGI and CICYT by project Contract Nos. BFM2001-2001, TIC2002-04528-C0201, and TIC2001-3163. Thanks to R. Pineda from Omicron Circuits, s.l. for the fabrication of the prototypes.

${ }^{1}$ V. G. Veselago, Sov. Phys. Usp. 10, 509 (1968).

${ }^{2}$ D. R. Smith and N. Kroll, Phys. Rev. Lett. 85, 2933 (2000).

${ }^{3}$ C. Caloz, C.-C. Chang, and T. Itoh, J. Appl. Phys. 90, 5483 (2001).

${ }^{4}$ D. R. Smith, W. J. Padilla, D. C. Vier, S. C. Nemat-Nasser, and S. Schultz, Phys. Rev. Lett. 84, 4184 (2000).

5 J. B. Pendry, A. J. Holden, D. J. Robbins, and W. J. Stewart, IEEE Trans. Microwave Theory Tech. 47, 2075 (1999).

${ }^{6}$ R. Marqués, J. Martel, F. Mesa, and F. Medina, Phys. Rev. Lett. 89, 183901 (2002).

${ }^{7}$ A. Grbic and G. V. Eleftheriades, J. Appl. Phys. 92, 5930 (2002).

${ }^{8}$ R. Marqués, F. Medina, and R. Rafii-El-Idrissi, Phys. Rev. B 65, 144440 (2002).

${ }^{9}$ R. Marqués, F. Mesa, J. Martel, and F. Medina, IEEE Trans. Antennas Propag. 5, 2572 (2003).

${ }^{10}$ G. V. Eleftheriades, O. Siddiqui, and A. K. Iyer, IEEE Microwave Wireless Components Lett. 13, 51 (2003).

${ }^{11}$ M. Pozar, Microwave Engineering (Addison-Wesley, Reading, MA), 1993. 